\title{
La caída de las cajas de ahorros españolas. Cuestión de rentabilidad, tamaño y estructura de propiedad* $^{*}$
}

\author{
SALVADOR CLIMENT SERRANO \\ Departamento de Análisis Económico, UNIVERSIDAD DE VALENCIA, ESPAÑA. E-mail: \\ salvador.climent@uv.es
}

\begin{abstract}
RESUMEN
En España entre 2010 y 2011 se ha producido una gran restructuración financiera, que ha llevado a la práctica desaparición de las Cajas de Ahorros mediante fusiones y posterior cambio de estructura de la propiedad convirtiéndose en bancos. Esta gran restructuración ha sido promovida por el Banco de España, como salida para que las entidades financieras subsistieran a la crisis que les afectaba.

Este trabajo analiza los bancos y cajas del sistema financiero español durante los cuatro años previos al comienzo de la reestructuración financiera del 2010. El objetivo es comprobar si existen diferencias en cuanto a rentabilidad por causas de tamaño y tipo de gobierno de la entidad (cajas de ahorros, bancos). Los resultados muestran que el incremento de tamaño y la bancarización auspiciadas por el Banco de España es una estrategia adecuada para resolver la actual crisis financiera.
\end{abstract}

Palabras clave: Cajas de ahorro, bancos, rentabilidad, fusiones.

\section{The Fall of Spanish Savings Banks. Question of Profitability, Size and Ownership Structure}

\begin{abstract}
In Spain between 2010 and 2011 there has been a major financial restructuring, which has led to the virtual disappearance of savings banks through mergers and subsequent change in ownership structure becoming banks. This major restructuring has been promoted by the Bank of Spain, as an outlet for that financial institutions subsisted to the crisis that affected them.

This paper analyzes the banks and savings banks the Spanish financial system during the four years prior to the beginning given the financial restructuring of 2010. The aim is to check whether there are differences in profitability for reasons of size and form of ownership of the entity (savings banks, banks). The results show that the increase in size and bankarization under the auspices by the Bank of Spain is an appropriate strategy for resolving the current financial crisis.
\end{abstract}

Keywords: Savings Banks, Banks, Profitability, Mergers.

Clasificación JEL: G21

* El autor agradece a dos revisores anónimos sus comentarios y sugerencias, que han permitido mejorar este trabajo.

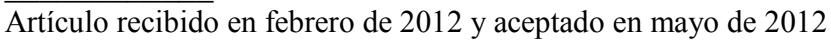

Artículo disponible en versión electrónica en la página www.revista-eea.net, ref. ə-30213 


\section{INTRODUCCIÓN}

En los años 2010 y 2011, se ha producido en España la mayor reestructuración financiera hasta la fecha. Esta reestructuración se ha centrado en las Cajas de Ahorros, ha venido motivada, en gran parte, por la crisis financiera internacional que empezó en el verano de 2007 con las hipotecas subprime y se acrecentó en el 2008 con la quiebra de Lehman Brothers. Si bien España no estaba muy contaminada por estos acontecimientos, sí que tenía otros problemas derivados de la gran inversión inmobiliaria que habían realizado las entidades financieras españolas durante los últimos años, además esta crisis internacional empeoró de forma sustancial la economía española llegando a niveles de desempleo por encima del $20 \%$. Todas estas circunstancias tuvieron su reflejo en las cuentas de las entidades financieras españolas, tanto en rentabilidad como en solvencia. La tasa de morosidad que era menor del 1\% en 2006 creció hasta el más del 8\% en 2012.

Ante estas circunstancias y para salvaguardar el sistema financiero español, el Banco de España instó a las entidades financieras a realizar una honda reestructuración financiera, basada en aumentar de tamaño y en cambiar la estructura de propiedad de las cajas de ahorros adoptando los principios de las sociedades anónimas igual que los bancos.

Este proceso se instrumentalizó, a instancias del Banco de España, mediante dos Reales Decretos Leyes. El 26 de junio de 2009 se aprobó el R.D.L 9/2009, sobre reestructuración bancaria y reforzamiento de los recursos propios de las entidades de crédito. Por el cual se apoyaban los procesos de integración viables, con la posibilidad de inyectar recursos económicos que computaran como recursos propios, reforzando de esta manera el coeficiente de solvencia de las entidades que lo solicitaban. El instrumento elegido para materializar esta operación fue las participaciones preferentes, que pueden ser convertibles en capital, por lo que computan como recursos básicos, estas participaciones tienen una rentabilidad mínima del $7.75 \%$ y se tienen que devolver en 5 o 7 años como máximo, en caso contrario se convertirían en capital. El 18 de febrero se aprobó el Real Decreto-ley 2/2011, de 18 de febrero, para el reforzamiento del sistema financiero, por el que se exige el $8 \%$ de capital básico a los bancos y el $10 \%$ a las Cajas de Ahorros que no coticen en bolsa.

Tras dos años a principios de 2012 de las 46 Cajas de Ahorros existentes, tan sólo dos seguían gestionando la activad financiera como Caja de Ahorros, entre las dos representan el $0.1 \%$ del total de recursos gestionado por las Cajas de Ahorros en 2009, último año antes de la restructuración.

En la Tabla $n^{\circ} 1$ vemos el proceso de integración que se ha producido: 
Tabla 1

Cajas de Ahorros españolas

\begin{tabular}{|c|c|c|c|c|c|}
\hline 1 & CAJA MADRID & \multirow{7}{*}{ SIP } & \multirow{7}{*}{ Bankia } & \multirow{7}{*}{1} & \multirow{7}{*}{1} \\
\hline 2 & BANCAJA & & & & \\
\hline 3 & LA CAJA DE CANARIAS & & & & \\
\hline 4 & LAIETANA & & & & \\
\hline 5 & AVILA & & & & \\
\hline 6 & SEGOVIA & & & & \\
\hline 7 & LA RIOJA & & & & \\
\hline 8 & LA CAIXA & \multirow{2}{*}{ FUSIÓN } & \multirow{2}{*}{ CaixaBank } & \multirow{7}{*}{$\begin{array}{c}\text { CaixaBank } \\
\text { (CaixaBank Absorbe a Banca } \\
\text { Cívica) }\end{array}$} & \multirow{7}{*}{2} \\
\hline 9 & GIRONA & & & & \\
\hline 10 & NAVARRA & \multirow{5}{*}{ SIP } & \multirow{5}{*}{$\begin{array}{l}\text { Banca Cívica } \\
\text { cotiza en bolsa }\end{array}$} & & \\
\hline 11 & CAJA BURGOS & & & & \\
\hline 12 & CAJA Canarias & & & & \\
\hline 13 & CAJA SOL & & & & \\
\hline 14 & GUADALAJARA & & & & \\
\hline 15 & CAJASTUR & \multirow{4}{*}{ SIP } & \multirow{4}{*}{ LiberBank } & \multirow{4}{*}{3} & \multirow{4}{*}{3} \\
\hline 16 & CCM & & & & \\
\hline 17 & EXTREMADURA & & & & \\
\hline 18 & CANTABRIA & & & & \\
\hline 19 & GRANADA & \multirow{4}{*}{ SIP } & \multirow{4}{*}{$\begin{array}{l}\text { BMN Banco } \\
\text { Mare Nostrum }\end{array}$} & \multirow{4}{*}{4} & \multirow{4}{*}{4} \\
\hline 20 & MURCIA & & & & \\
\hline 21 & PENADES & & & & \\
\hline 22 & SA NOSTRA & & & & \\
\hline 23 & CAI & \multirow{3}{*}{ SIP } & \multirow{3}{*}{ Banco Caja Tres } & \multirow{4}{*}{$\begin{array}{c}\text { Banco Ibercaja } \\
\text { (Ibercaja absorbe a Caja Tres) }\end{array}$} & \multirow{4}{*}{5} \\
\hline 24 & BADAJOZ & & & & \\
\hline 25 & CIRCULO BURGOS & & & & \\
\hline 26 & IBERCAJA & & Banco Ibercaja & & \\
\hline 27 & BBK & & & & \\
\hline 28 & CAJASUR & SID & Banco Bilbao & 6 & 6 \\
\hline 29 & KUTXA & SIP & Bizkaia Kutxa & 6 & 6 \\
\hline 30 & VITAL KUTXA & & & & \\
\hline 31 & UNICAJA & & & & \\
\hline 32 & JAEN & Fusión & Banco Ceiss & Unicaja banco & \\
\hline 33 & CAJA DUERO & & & Unicaia ahsorhe a Caia Fsnaña & 7 \\
\hline 34 & CAJA ESPAÑA & Fusión & Unicaja Banco & Unıcaja absorbe a Caja Espana & \\
\hline 35 & ONTINYENT & & Caja de ahorros & 8 & 8 \\
\hline 36 & POLLENSA & & Caja de ahorros & 9 & 9 \\
\hline 37 & CECA & & & & 10 \\
\hline 38 & CAM & & ervenida - Absorbic & a por Banco Sabadell & \\
\hline 39 & SABADELL & & & & \\
\hline 40 & TARRASA & FUSIÓN & Unin & Absorbida por el BBVA & \\
\hline 41 & MANLLEU & & & & \\
\hline 42 & CATALUNYA & & & & \\
\hline 43 & TARRAGONA & FUSIÓN & & aluya Caixa.Intervenida & \\
\hline 44 & MANRESA & & & & \\
\hline 45 & GALICIA & FUSION & & ivagalicia Interyon & \\
\hline 46 & CAIXANOVA & FUSION & & Ixagalicia. Intervenida & \\
\hline
\end{tabular}

Fuente: Elaboración propia. 
De las 46 entidades que existían en 2009, se han reducido a 7 grupos, las dos cajas pequeñas y la CECA. Con dos Cajas intervenidas que en estos momentos aún no se sabe el desenlace.

Todas las cajas, excepto las dos mencionadas, han cedido sus activos y pasivos a bancos y funcionan como tales. De los cuales tres cotizan en bolsa. CaixaBank, Bankia y Banca Cívica absorbida por CaixaBank. Las dos que se mantienen como cajas tradicionales son Caixa Ontinyent y Pollensa, estas dos significa el $0.1 \%$ del total de recursos gestionados. Existen otros grupos de cajas que han sido intervenidas por el Banco de España o que el FROB ha tomado su gestión, son la CAM que ya ha sido absorbida por el Banco Sabadell, UNIM adquirida por el BBVA, y CatalunyaCaixa y NovaCaixagalicia.

Este trabajo se organiza en seis apartados. En el primero se ha realizado una introducción sobre el estado de la cuestión. En el segundo se presentan los objetivos. En el tercero se expone la metodología utilizada. En el cuarto se presenta la evolución de la cuenta de pérdidas y ganancias de las entidades estudiadas en el periodo estudiado. En el quinto se exponen los resultados de los tres grandes bloques, ingresos, gastos y rentabilidad. En el sexto y último se expone el resultado final y las conclusiones.

\section{OBJETIVOS}

Con este proceso de reestructuración han desaparecido las cajas de ahorros españolas como entidades financieras, tal como habían desarrollado su función desde el siglo XIX. Este proceso ha llevado a las cajas, además de fusionarse con el objetivo de ganar en tamaño, a transformase en bancos.

Este acontecimiento es lo suficientemente importante para investigar si el camino marcado por el Banco de España, para solucionar la crisis financiera es adecuado, tanto en el objetivo marcado de incremento de tamaño como el de bancarización, que ha llevado a la desaparición de la gestión financiera de las cajas de ahorros españolas.

Se han marcado dos objetivos, el primero es estudiar si en la gestión de la crisis que ha conducido a esta reestructuración, los bancos han gestionado mejor que las cajas y obtienen una mayor rentabilidad que les permite afrontar con mayor solvencia la actual crisis. El segundo es comprobar si el tamaño importa, ya que a instancias del Banco de España las entidades han tenido que fusionarse para ganar en tamaño, lo que debe de implicar que las entidades grandes deben de tener una mayor rentabilidad que las pequeñas.

Respecto al incremento de tamaño de las entidades, en otros estudios realizados como los de Gilbert, (1984); Smirlock, (1985); Hernández, (1991); Gual y Apellaniz et al, (1995); Martínez Arias, (1998); Arévalo et al, (2002); Colarte Morando et al, (2006); Guerrero Mora, (2009); Marín et al, (2008); tratan el 
tema de las fusiones bancarias y la relación entre el tamaño y la rentabilidad. La principal conclusión que obtienen es que no siempre se gana eficiencia y rentabilidad tras las fusiones, incluso en algunas veces se pierde.

Entre las ventajas de aumentar en tamaño está el facilitar el control y supervisión de las autoridades monetarias, la mayor diversificación del riesgo, la eliminación de las entidades menos competitivas quedando las más rentables y solventes, la mayor facilidad de acceso a los mercados mayoristas y el convertirse, por su tamaño, en entidades sistémicas con lo que serian demasiado grandes para quebrar (Palomo y Sanchis, 2010), (Vives, 2011), (González, 2011).

Respecto a la diferencia entre bancos y cajas, estudios como los de Coello Aranda, (1994); Pastor, (1995); Maudos y Pérez, (2003); y tratan sobre la diferencia entre las cajas y los bancos. El trabajo de Azofra Palenzuela (2004), estudia el gobierno de las cajas de ahorros y su influencia en la gestión de las mismas, esta diferenciación puede ser una de las causas de la divergencia en rentabilidad entre bancos y cajas.

Esta opinión es mantenida por otros autores, por ejemplo, Jensen y Meckling (1976) apuntaban ya en la década de los setenta del siglo pasado que la forma jurídica que adoptaban las empresas podría influir es su rentabilidad. En cuanto a las cajas de ahorros españolas, Fonseca et al (2011) establecen que la ausencia de propietarios accionistas puede motivar diferencias entre cajas y bancos en cuanto tipo impositivo efectivo. Estas diferencias pueden estar motivadas por el destino de los beneficios y por la forma de elegir los órganos de gobierno en las cajas de ahorros. Por una parte, los beneficios no son destinados a ningún grupo de interés decisor de la inversión y financiación, si no que se dedican a la obra benéfico-social o a reservas. Mientras, por otra, la elección de los órganos de gobierno está enormemente politizada, lo que puede acarrear un mayor descontrol directivo que puede derivar en un menor interés en la maximización de la rentabilidad de las sociedades, ya que no rige el pago de dividendos, y se priorizan otros objetivos, marcados por los que han elegido a los miembros del gobierno de las cajas, asambleas y consejos de administración.

Los resultados obtenidos puedes discrepar con los estudiados en la literatura existente, ya que, en el proceso actual, existen dos diferencias sustanciales sobre las otras etapas de fusiones. La primera en cuanto a los aspectos geográficos, la mayor parte de fusiones anteriores han sido entre entidades de la misma zona geográfica, por lo que la duplicidad de oficinas era la nota característica. En este caso se han producido muchas fusiones interregionales por lo que esta desventaja ha disminuido. En segundo lugar y que se ha producido por primera vez, además de fusionarse las entidades han cambiado la estructura de la propiedad y se han convertido en bancos, quedando en la caja de ahorros tan sólo la gestión de la obra social que se llevará a cabo con los dividendos que aporte su participación en el banco. 


\section{METODOLOGÍA}

\subsection{Muestra}

Todas las cajas de ahorro y los 13 bancos más importantes. Del conjunto de todas las cajas hemos suprimido a CCM, que fue intervenida a principios de 2009 y a la CECA. La muestra supone más del $98 \%$ del total del sistema financiero comercial español.

La clasificación por tamaño la hemos hecho de la siguiente manera ${ }^{1}$ :

- Muy grandes. Entidades con un activo mayor a 135.000 millones de euros, son 2 cajas y dos bancos, Caja Madrid, La Caixa, BBVA y Santander.

- Grandes. Entidades en las que su activo está entre los 45.000 y 134.999 millones de euros. Las entidades que lo conforman son: Bancaja, Banesto, Banco Sabadell, CAM, Bankinter, Caja Catalunya, Caja de Ahorros de Galicia e Ibercaja. Cinco cajas y tres bancos.

- Medianas. Activo entre 15.000 y 44.999 millones de euros. Son: Barclays Bank, Unicaja, Banco Pastor, Caixa Nova, BBK, Caja Sol, Caja España, Caixa Penedés, Banco de Valencia, Caja Murcia, Caja Duero, KUTXA, Caja Navarra, Cajasur y CajAstur. Doce cajas y tres bancos.

- Pequeñas. Entidades que su activo está entre los 5.000 millones y los 14.999 millones. Son: SaNostra, Caja Canarias (Caja General), Caja Granada, Caixa Sabadell, Caja Burgos (Municipal), Banca March, Caixa Tarrassa, CAI, Banco Guipuzcoano, Caja Cantabria, Caixa Tarragona, La Caja de Canarias, (Insular), Caixa Laietana, Vital Kutxa, Caixa Girona, Caja Extremadura, Caja Ávila, Caixa Manresa, Caja Segovia, Caja Círculo, (Burgos). Dos bancos y dieciocho cajas.

- Muy pequeñas. Entidades con un activo menor de 5.000 millones de euros. Son: Banco Gallego, Caja Badajoz, Caja Rioja, Caja Manlleu, Caja Guadalajara, Caixa Ontinyent, Caja Jaén, Banco Pueyo, y Caixa Pollensa. Siete cajas y dos bancos.

\subsection{Modelo}

El modelo que se ha implementado se basa en el análisis de la cuenta de pérdidas y ganancias de las entidades financieras, mediante la cual se han construido dieciocho indicadores divididos en tres bloques Seis de ingresos, ocho de gastos y cuatro de rentabilidad. Todos los indicadores se elaboran aplicando diferencia de medias, obteniendo la diferencia de la media de todas las entida-

\footnotetext{
${ }^{1}$ La clasificación por tamaño se ha realizado teniendo en cuenta el tamaño de las entidades que lo conforman. Partiendo de las que se consideran muy grandes con una activo superior a 135.000 millones y dividendo por tres cada vez hasta llegar a las muy pequeñas.
} 
des respecto de la media del grupo de comparación. En todos también se incorpora la tendencia tomando los valores de los cuatro años y ponderándose por inmediatez.

\subsubsection{Indicadores de ingresos ${ }^{2}$}

A.- IMi.- Margen de interés

El primer indicador de ingresos es el margen de interés o de intermediación. Informa sobre los intereses netos que percibe la entidad. Este indicador se ha construido sobre la base de la diferencia entre los intereses que la entidad cobra por sus activos remunerados y los intereses que retribuye a sus pasivos.

Su formulación es la siguiente:

$$
\text { IMi }=\left(\left(i_{h 06}+i_{h 07} * 2+i_{h 08} * 3+i_{09} * 4\right) / 10\right)-\left(\left(i_{\mu 06}+i_{\mu 07} * 2+i_{\mu 08} * 3+i_{\mu 09} * 4\right) / 10\right)
$$

Siendo:

$i_{h x x}$ : Diferencia entre las tasas de interés cobradas y pagadas del año xx del grupo $h$.

$i_{\mu x x}$ : Diferencia entre las tasas de intereses cobradas y pagadas del año xx de la media de todas las entidades.

B.- IRc. Rendimientos instrumentos de capital

El segundo indicador mide los rendimientos de capital de las entidades financieras analizadas. Se construye de forma similar al anterior:

$$
\begin{aligned}
& I R c=\left(\left(R c_{h 06}+R c_{h 07} * 2+R c_{h 08} * 3+R c_{h 09} * 4\right) / 10\right)- \\
&\left(\left(R c_{\mu 06}+R c_{\mu 07} * 2+R c_{\mu 08} * 3+R c_{\mu 09} * 4\right) / 10\right)
\end{aligned}
$$

Siendo:

$R c_{h x x}$ : Tasa de rendimiento medio de las inversiones en capital de las entidades del grupo $\mathrm{h}$.

$R c_{\mu x x}$ : Tasa de rendimiento medio de las inversiones en capital de todas las entidades.

C.- IRp. Rendimiento participadas

\footnotetext{
${ }^{2}$ Los indicadores elegidos son los que determinan la rentabilidad de las entidades financieras, todas las cuentas de ingresos y de gastos que conforman la cuenta de pérdidas y ganancias y que han sido utilizados en los estudios previos examinados en la literatura. Son los indicadores que se utilizan habitualmente en el sector y que reflejan la mayor parte de los aspectos relevantes en el negocio bancario.
} 
Este indicador mide los rendimientos de las inversiones en las empresas participadas.

$$
\operatorname{Irp}=\left(\begin{array}{l}
\left.\left(R p_{h 06}+R p_{h 07} * 2+R p_{h 08} * 3+R p_{h 09} * 4\right) / 10\right)- \\
\left.\left(R p_{\mu 06}+R p_{\mu 07} * 2+R p_{\mu 08} * 3+R p_{\mu 09} * 4\right) / 10\right)
\end{array}\right.
$$

Siendo:

$R p_{h x x}$ : Tasa media de rentabilidad de las empresas participadas de las empresas del grupo de comparación h.

$R p_{\mu x x}$ : Tasa media de rentabilidad de las empresas participadas del total de empresas

D.- Ic.- Comisiones

Este indicador mide los ingresos por comisiones netas, (cobradas menos pagadas)

$$
\left.I c=\left(\left(c_{h 06}+c_{h 07} * 2+c_{h 08} * 3+c_{09} * 4\right) / 10\right)-\left(c_{\mu 06}+c_{\mu 07} * c+c_{\mu 08} * 3+c_{\mu 09} * 4\right) / 10\right)
$$

Siendo

$C_{h x x}$ : Tasa media de las comisiones del año xx del grupo de comparación $\mathrm{h}$.

$C_{\mu x x}$ : Tasa media de las comisiones del año xx del total de la muestra.

E.- IRop. Resultado de operaciones financieras

Este indicador mide la rentabilidad de la cuenta de operaciones financieras. Como ejemplo de algunos ingresos contabilizados en esta cuenta están la compra de títulos propios con beneficio o los resultados de la venta de títulos de la cartera especulativa. Estos ingresos se suelen forzar cuando las entidades tienen necesidades de aflorar plusvalías, como por ejemplo en años de bajos beneficios ordinarios o por incrementos de necesidades de capital.

$$
I R o p=\left(\begin{array}{l}
\left.\left(\operatorname{Rop}_{h 06}+\operatorname{Rop}_{h 07} * 2+\operatorname{Rop}_{h 08} * 3+\operatorname{Rop}_{h 00} * 4\right) / 10\right) \\
\left.\left.\operatorname{Rop}_{\mu 06}+\operatorname{Rop}_{\mu 07} * 2+\operatorname{Rop}_{\mu 08} * 3+\operatorname{Rop}_{\mu 09} * 4\right) / 10\right)
\end{array}\right.
$$

Siendo:

$R o p_{h x x}$ : Rentabilidad sobre activo del año xx de los resultados de las operaciones financieras del grupo de comparación $h$.

$R o p_{\mu x x}$ : Rentabilidad sobre activo del año xx de los resultados de las operaciones financieras del total de la muestra.

F.- IMb.- Margen bruto

En este caso se evalúa el margen bruto. 


$$
I M b=\left(\begin{array}{l}
\left.\left(M b_{h 06}+M b_{h 07} * 2+M b_{h 08} * 3+M b_{h 09} * 4\right) / 10\right) \\
\left(M b_{\mu 06}+M b_{\mu 07} * 2+M b_{\mu 08} * 3+M b_{\mu 09} * 4\right) / 10
\end{array}\right)
$$

Siendo:

$M b_{h x x}$ : Media del margen bruto del año xx de grupo de comparación $\mathrm{h}$.

$M b_{\mu x x}$ : Media del margen bruto del año xx de todas las entidades de la muestra.

\subsubsection{Indicadores de gastos}

En los indicadores a1, a2, b, c, y e, que son gastos, el indicador se construye como la media de todas las entidades menos la media del grupo de comparación, de esta manera si la media de los gastos del grupo de comparación es mayor que la media de todas las entidades obtendremos un resultado negativo. De esta forma un resultado más positivo (o menos negativo) denota una entidad con mejores resultados, al tener menos gastos de administración, a1 y a2; menos pérdidas por deterioro de inversiones crediticias, $b$; menos pérdidas por otros instrumentos financieros valorados a valor razonable, c; menos pérdidas por deterioro del resto de activos.

En el resto de apartados d, f, y g, que ha pesar de estar en el bloque de gastos siguiendo la estructura de la cuenta de pérdidas y ganancias, un saldo mayor significa mejores resultados, se construyen de la misma manera que en el apartado de ingresos. De esta forma un resultado más positivo (o menos negativo) denota una entidad con mejores resultados, al tener mayores resultados de explotación.

\section{A1.-IEa.- Indicador eficiencia sobre activo. (Gastos de administración sobre activo)}

Este indicador mide la eficiencia de las entidades, ya que se calcula como el cociente entre los gastos de administración y el total del activo. Nos indica el porcentaje gastos de administración que hace falta para gestionar el activo.

$$
\begin{aligned}
I E a= & \left.\left(E a_{\mu 06}+R b_{\mu 07} * 2+E a_{\mu 08} * 3+M b_{\mu 09} * 4\right) / 10\right)- \\
& \left(\left(E a_{h 06}+E a_{h 07} * 2+E a_{h 08} * 3+E a_{h 09} * 4\right) / 10\right)
\end{aligned}
$$

Siendo:

$E a_{\mu x x}$ : Cociente entre los gastos de administración y el activo remunerado del total de empresas de la muestra del año xx.

$E a_{h x x}$ : Cociente entre los gastos de administración y el activo remunerado del grupo de comparación del año xx. 
A2.- Indicador de eficiencia sobre margen bruto

Este indicador esta basado en el ratio de eficiencia de las entidades, gastos de administración dividido entre el margen bruto.

$$
\begin{aligned}
I E M b= & \left(\left(E M b_{\mu 06}+E M b_{\mu 07} * 2+E M b_{\mu 08} * 3+E M b_{\mu 09} * 4\right) / 10\right)- \\
& \left(\left(E M b_{h 06}+E M b_{h 07} * 2+E M b_{h 08} * 3+E M b_{h 09} * 4\right) / 10\right)
\end{aligned}
$$

Siendo:

$E M b_{\mu x x}$ : Ratio de eficiencia, gastos de administración entre margen bruto, de todas las entidades de la muestra del año xx.

$E M b_{h x x}$ : Ratio de eficiencia, gastos de administración entre margen bruto, de las entidades del grupo de comparación del año xx.

B. IPic. Pérdidas por deterioro de inversiones crediticias

Este indicador mide los impagados de las inversiones en créditos a clientes.

$$
\begin{aligned}
I P i c= & \left.\left(P i c_{\mu 06}+P i c_{\mu 07} * 2+P i c_{\mu 08} * 3+P i c_{\mu 09} * 4\right) / 10\right)- \\
& \left(\left(P i c_{h 06}+P i c_{h 07} * 2+P i c_{h 08} * 3+P i c_{h 09} * 4\right) / 10\right)
\end{aligned}
$$

Siendo:

$P i c_{\mu x x}$ : Dotaciones del año $\mathrm{xx}$ de la media de todas las entidades de la muestra.

$P i c_{h x x}$ : Dotaciones del año xx de la media de las entidades del grupo de comparación.

C.- IPpyg Pérdidas de otros activos con cambios en P y G

Este indicador mide las dotaciones que se practican sobre los activos financieros disponibles para la venta.

$$
\begin{aligned}
I \text { Ppyg }= & \left(\left(\text { Ppyg }_{\mu 06}+\text { Ppyg }_{\mu 07} * 2+\text { Ppyg }_{\mu 08} * 3+\text { Ppyg }_{\mu 09} * 4\right) / 10\right)- \\
& \left(\left(\text { Ppyg }_{h 06}+\text { Ppyg }_{h 07} * 2+\text { Ppyg }_{h 08} * 3+P p y g_{h 09} * 4\right) / 10\right)
\end{aligned}
$$

Siendo:

$\operatorname{Ppyg}_{\mu x x}$ : Ratio media entre las dotaciones y los activos financieros disponibles para la venta del año xx de todas las entidades de la muestra.

Ppyg $_{h x x}$ : Ratio media entre las dotaciones y los activos financieros disponibles para la venta del año xx de las entidades del grupo de comparación.

D.-IRe. Resultados de explotación 
Este indicador mide los resultados de explotación, en este caso lo construimos de manera que cuanto mayor sea el beneficio que obtenga la entidad, la puntuación sea mayor.

$$
I R e=\left(\begin{array}{l}
\left.\left(\operatorname{Re}_{h 06}+\operatorname{Re}_{h 07} * 2+\operatorname{Re}_{h 08} * 3+\operatorname{Re}_{h 09} * 4\right) / 10\right)- \\
\left.\left(\operatorname{Re}_{\mu 06}+\operatorname{Re}_{\mu 07} * 2+\operatorname{Re}_{\mu 08} * 3+\operatorname{Re}_{\mu 09} * 4\right) / 10\right)
\end{array}\right.
$$

Siendo:

$R e_{h x x}$ : Ratio media del año xx entre el margen de explotación y los activos de las entidades del grupo de comparación.

$R e_{\mu x x}$ : Ratio media del año xx entre el margen de explotación y los activos de todas las entidades de la muestra.

E.- IPra. Pérdidas en resto de activos

Este indicador mide las dotaciones por pérdidas en el resto de activos. Prácticamente esta compuesto por las dotaciones sobre las minusvalías de las entidades participadas. Por eso utilizamos como base para obtener la ratio el valor de las entidades participadas. Lo construimos de la forma habitual para los gastos.

$$
\begin{array}{r}
I \text { Pra }= \\
\left.\left(\operatorname{Pra}_{\mu 06}+\operatorname{Pr}_{\mu 07} * 2+\operatorname{Pra}_{\mu 08} * 3+\operatorname{Pra}_{\mu 09} * 4\right) / 10\right)- \\
\left(\left(\operatorname{Pra}_{h 06}+\operatorname{Pra}_{h 07} * 2+\operatorname{Pra}_{h 08} * 3+\operatorname{Pr}_{h 09} * 4\right) / 10\right)
\end{array}
$$

Siendo:

$\operatorname{Pr} a_{\mu x x}$ : Ratio media del año xx de las dotaciones por el deterioro del resto de activos entre las participadas del total de las entidades de la muestra.

$\operatorname{Pr} a_{h x x}$ : Ratio media del año xx de las dotaciones por el deterioro del resto de activos entre las participadas de las entidades del grupo de comparación.

F.- IGre. Ganancias en la baja de activos no corrientes

Este indicador mide los resultados, (ganancias o pérdidas) en la baja de los activos no clasificados como no corrientes en venta. Estos activos son el inmovilizado material y las participadas. Es similar a lo que se conocía como resultados extraordinarios. En este caso sólo disponemos de información de los dos últimos años.

Construimos el indicador igual que el de resultado de explotación.

$$
\text { IGre } \left.\left.=\left(\text { Gre }_{h 08} * 3+\operatorname{Gre}_{09} * 7\right) / 10\right)-\left(\operatorname{Gre}_{\mu 08} * 3+\text { Gre }_{\mu 09} * 7\right) / 10\right)
$$

Siendo: 
Gre $_{h x x}$ : Ratio media entre los resultados de esta cuenta del año xx y la suma de las participadas más el inmovilizado material de las entidades del grupo de comparación.

Gre $_{\mu x x}$ : Ratio media entre los resultados de esta cuenta del año xx y la suma de las participadas más el inmovilizado material del total de entidades.

G.- IGAncv. Ganancias en la venta de los activos no corrientes en venta

Este indicador mide los resultados de la venta de los activos no corrientes en venta no clasificados como operaciones interrumpidas. En esta cuenta se contabiliza la venta de los inmuebles adjudicados, las dotaciones de deterioro de los mismos y los resultados y el deterioro de los activos considerados como inversiones estratégicas.

Sólo disponemos de información de dos años.

\subsubsection{Indicadores de rentabilidad}

El tercer grupo de indicadores son los relacionados con la rentabilidad.

A.- IROAe. Rentabilidad sobre activo de la entidad

Nos mide la rentabilidad sobre los activos de la entidad individual.

$$
\begin{gathered}
I R O A e=\left(\left(R O A e_{h 06}+R O A e_{h 07} * 2+R O A e_{h 08} * 3+R O A e_{h 09} * 4\right) / 10\right)- \\
\left(\left(R O A e_{\mu 06}+R O A e_{\mu 07} * 2+R O A e_{\mu 08} * 3+R O A e_{\mu 09} * 4\right) / 10\right)
\end{gathered}
$$

Siendo:

$R O A e_{h x x}$ : Rentabilidad de la entidad individual sobre activos del año xx de las entidades que conforman el grupo de comparación.

$R O A e_{\mu x x}$ : Rentabilidad de la entidad individual sobre activos del año xx de todas las entidades de la muestra.

B.- IROEe. Rentabilidad sobre fondos propios

Mide la rentabilidad sobre los fondos propios de la entidad individual.

$$
\begin{aligned}
I R O E e= & \left(\left(R_{R E e_{h 06}}+\text { ROEe }_{h 07} * 2+\text { ROEe }_{h 08} * 3+\text { ROEe }_{h 09} * 4\right) / 10\right)- \\
& \left.\left(\text { ROEe }_{\mu 06}+\text { ROEe }_{\mu 07} * 2+\text { ROEe }_{\mu 08} * 3+\text { ROEe }_{\mu 09} * 4\right) / 10\right)
\end{aligned}
$$

Siendo:

$R O E e_{h x x}$ : Rentabilidad de la entidad individual sobre fondos propios del año xx de la las entidades que conforman el grupo de comparación. 
$R O E e_{\mu x x}$ : Rentabilidad de la entidad individual sobre fondos propios del año xx de todas las entidades de la muestra.

C. IROAg. Rentabilidad sobre activos del grupo de sociedades

Nos mide la rentabilidad sobre los activos del grupo de sociedades.

$$
\begin{array}{r}
I R O A g=\left(\left(R O A g_{h 06}+R O A g_{h 07} * 2+R O A g_{h 08} * 3+R O A g_{h 09} * 4\right) / 10\right)- \\
\left.\left(R O A g_{\mu 06}+R O A g_{\mu 07} * 2+R O A g_{\mu 08} * 3+R O A g_{\mu 09} * 4\right) / 10\right)
\end{array}
$$

Siendo:

$R O A g_{h x x}$ : Rentabilidad del grupo de sociedades sobre activos del año xx de las entidades que conforman el grupo de comparación.

$R O A g_{\mu x x}$ : Rentabilidad del grupo de sociedades sobre activos del año xx de todas las entidades de la muestra.

D.- IROEg -Rentabilidad sobre fondos propios del grupo de sociedades

Nos mide la rentabilidad sobre los fondos propios del grupo de sociedades.

$$
I R O E g=\left(\begin{array}{l}
\left.\left(R O E g_{h 06}+R O E g_{h 07} * 2+R O E g_{h 08} * 3+R O E g_{h 09} * 4\right) / 10\right)- \\
\left.\left(R O E g_{\mu 06}+R O E g_{\mu 07} * 2+R O E g_{\mu 08} * 3+R O E g_{\mu 09} * 4\right) / 10\right)
\end{array}\right.
$$

Siendo:

$R O E g_{h x x}$ : Rentabilidad del grupo de sociedades sobre fondos propios del año xx de las entidades que conforman el grupo de comparación.

$R O E e_{\mu x x}$ : Rentabilidad del grupo de sociedades sobre fondos propios del año xx de todas las entidades de la muestra.

\section{GESTIÓN DE LA CRISIS}

Los indicadores anteriores durante la crisis han evolucionado de la siguiente manera.

\subsection{Ingresos}

\section{A.- Margen de intermediación}

El margen de intermediación aumentó en el periodo estudiado, pasando de un diferencial del $1.20 \%$ entre intereses cobrados y pagados al $1.89 \%$.

Los bancos obtienen un margen mayor que las cajas durante todo el periodo, pero sobre todo el en 2009 que es de $2.40 \%$ por $1.39 \%$ de las cajas.

En cuanto a tamaño las muy pequeñas son las que tienen un diferencial mayor, la media ponderada por inmediatez de los cuatro años es del 1.94\%, sin embargo el siguiente grupo son las muy grandes con un diferencial del $1.62 \%$, 
después desciende hasta el $1.45 \%$ de las pequeñas, el $1.32 \%$ de las medianas, y el diferencial menor lo tienen las grandes con un $1.25 \%$ de media ponderada por inmediatez en los cuatro ejercicios.

\section{B.-Rendimientos instrumentos de capital}

Los rendimientos de los instrumentos de capital permanecieron constantes, incluso aumentaron de forma moderada, en 2006 eran el 3.65\% del capital invertido y en 2009 eran el $4.61 \%$.

En cuanto a la diferencia de los rendimientos entre cajas y bancos son bastante importantes, ya que los bancos obtuvieron una rentabilidad media ponderada del $5.68 \%$ y las cajas del $3.57 \%$.

Sí que hay relación entre el tamaño y la rentabilidad, así el grupo que mayor rendimiento obtienen es el de las muy grandes, con un rendimiento medio ponderado del $5.05 \%$, las grandes un poco menos, el $3.83 \%$, las medianas el $3.44 \%$, las pequeñas el $2.61 \%$ y las muy pequeñas el $3.27 \%$.

\section{C.-Resultado de entidades valoradas por el método de la participación}

Los resultados que obtienen estas entidades de sus sociedades participadas han tenido un gran descenso. En 2006 obtenían una rentabilidad del $12.86 \%$ y en 2009 del $3.02 \%$.

Aunque tanto en bancos como en cajas la rentabilidad de estas empresas ha disminuido de forma considerable, los bancos obtienen mejor rentabilidad que las cajas, así la rentabilidad media ponderada de los bancos es del $8.36 \%$ y la de las cajas del $6.06 \%$.

Por tamaño también existe una relación entre tamaño y rentabilidad, aunque con una excepción en las entidades pequeñas, que son las que mayor rentabilidad obtienen con un $11.65 \%$. A partir de estas entidades sigue una relación entre tamaño y rentabilidad, las muy grandes obtuvieron un $7.97 \%$ por término medio ponderado, las grandes el $4.42 \%$ las medianas el $3.39 \%$ y las muy pequeñas un rentabilidad negativa del $2.65 \%$.

\section{D.- Comisiones}

El porcentaje que cobran las entidades financieras de este estudio por comisiones a sus clientes han permanecido constantes en el periodo analizado, con una pequeña reducción. En 2006 era el $0.68 \%$ de los activos remunerados y en 2009 el $0.62 \%$.

Tanto bancos como cajas han disminuido de forma moderada estos ingresos. Pero los bancos obtienen unas comisiones muy superiores a las cajas. Los bancos obtuvieron una rentabilidad media ponderada de comisiones del $0.78 \%$ sobre los activos remunerados, mientras que las cajas era del $0.41 \%$. 
También existe correlación entre comisiones y tamaño, sobre todo en las entidades de mayor tamaño. Las muy grandes obtienen el $0.75 \%$, las grandes el $0.46 \%$, las muy pequeñas el $0.41 \%$, las pequeñas el $0.39 \%$ y las que menos, las medianas con el $0.39 \%$.

\section{E.-Resultados de operaciones financieras}

Esta cuenta, que entre otras partidas, contabiliza los resultados de la venta de activos financieros disponibles para la venta (cartera especulativa), por ejemplo mediante la compra de activos propios con beneficios, ha permanecido casi igual en este periodo era el $0.31 \%$ de los activos en 2006 y en 2009 era el $0.28 \%$.

No existe mucha diferencia entre cajas y bancos, en los bancos la media ponderada era del $0.30 \%$ y en las cajas del $0.28 \%$.

Por tamaño no existe correlación entre tamaño y rendimiento de esta cuenta, así las medianas obtienen un $0.40 \%$, las muy grandes el $0.28 \%$, las pequeñas el $0.24 \%$, las grandes el $0.21 \%$ y las muy pequeñas el $0.12 \%$.

\section{F.- Margen bruto}

La última variable de ingresos estudiada es el margen bruto. Durante este período ha aumentado de forma moderada, en 2006 era el $2.81 \%$ de los activos y en 2009 era el 3.03\%.

Los bancos obtienen bastante mejor rentabilidad que las cajas. Así la media ponderada del margen de los bancos es el $3.18 \%$ de sus activos y el de las cajas es el $2.50 \%$.

Por tamaño las que obtienen un mayor margen bruto son las muy grandes con el $3.19 \%$, las muy pequeñas obtienen un $2.83 \%$, las medianas el $2.74 \%$, las pequeñas el $2.62 \%$ y las que menor rentabilidad obtienen en el margen bruto son las grandes, el $2.43 \%$.

\subsection{Gastos}

\section{A.- Gastos de administración}

Los gastos de administración aumentaron de forma moderada durante el periodo estudiado, en 2006 eran el 1.16\% del activo remunerado y en 2009 eran el $1.20 \%$.

Estos gastos son mayores en términos relativos en los bancos que en las cajas, ya que en los bancos eran el $1.26 \%$ de los activos remunerados y en las cajas eran el $1.06 \%$.

Por tamaño las que más recursos utilizan para gestionar el activo remunerado son las muy pequeñas en las que estos gastos son el $1.66 \%$ de los activos remu- 
nerados, en las muy grandes son el $1.23 \%$, en las medianas el $1.19 \%$ en las pequeñas el $1.11 \%$ y las que tienen una ratio menor son las grandes, el $0.92 \%$.

\section{B.- Pérdidas por deterioro de inversiones crediticias}

Las dotaciones por deterioros de las inversiones en créditos a los clientes han aumentando de forma considerable, en 2006 eran el $0.43 \%$ de las inversiones crediticias y en 2009 eran el 1.21\%, casi el triple. Además no han dejado de crecer en 2010 y 2011.

En los bancos afloraron primero, ya que la media ponderada de estas dotaciones es del $0.94 \%$ de las inversiones crediticias y en las cajas eran del $0.78 \%$.

Por tamaño en las muy grandes eran el $0.97 \%$, en las medianas el $0.89 \%$, en las pequeñas el $0.73 \%$, en las grandes el $0.71 \%$ y las que menos eran las muy pequeñas con el $0.56 \%$.

\section{C.- Pérdidas de otros instrumentos financieros no valorados a valor razonable con cambios en $P$ y $G$.}

En esta cuenta en la que se contabilizan las dotaciones por deterioro de los activos financieros disponibles para la venta (Cartera especulativa). Por ejemplo disminución del valor del mercado de las acciones que posee la entidad en la cuenta de activos financieros disponibles para la venta. También han aumentado de forma considerable, estas dotaciones eran el $0.36 \%$ de los mismos en 2006 y en 2009 eran el $0.66 \%$ el doble.

Estas dotaciones son mayores en los bancos que en las cajas, en los bancos la media ponderada por inmediatez en los cuatro años eran el $0.78 \%$ de los activos financieros disponibles para la venta y en las cajas el $0.52 \%$.

No existe relación directa por tamaño, ya que las que más recursos pierden en este concepto son las grandes con el $1.16 \%$, después las medianas con el $0.74 \%$, las muy pequeñas con el $0.66 \%$, las muy grandes el $0.41 \%$ y por último las pequeñas el $0.41 \%$.

\section{D.- Resultado de explotación}

El resultado de explotación respecto al activo ha disminuido de forma considerable. En 2006 el resultado de explotación era el 1.06\% de activo y en 2009 era el $0.76 \%$.

Los bancos obtienen un mejor resultado que las cajas, la media ponderada de los cuatro años es el $1.00 \%$ del activo y en las cajas es del $0.67 \%$.

Por tamaño, en este caso sí que existe una correlación, cuanto mayor es la entidad mayor es el resultado de explotación. En las muy grandes es el 1.02\% 
del activo, en las grandes el $0.71 \%$, en las medianas el $0.62 \%$, en las muy pequeñas el $0.52 \%$, y tan sólo en las pequeñas se rompe esta tendencia, ya que es el $0.79 \%$.

\section{E.- Pérdidas por deterioro del resto de activos}

En esta cuenta se contabilizan las dotaciones para pérdidas en el fondo de comercio, el deterioro de las acciones de las participadas y el deterioro en existencias (solares, promociones en curso, construcciones y edificios). La evolución en los dos años últimos ${ }^{3}$ es muy importante, pasa de ser el $9.08 \%$ de las participadas, en 2008 al $19.97 \%$ en 2009 .

El aumento es muy importante tanto en bancos como en cajas, pero en los bancos la dotación es mayor, el $22.69 \%$ de saldo de las participadas, y en las cajas es el 5.07\% como media ponderada de los dos años.

Por tamaño no existe relación entre tamaño y porcentaje de estas dotaciones.

\section{F.- Ganancias (pérdidas) en la baja de activos no clasificados como no corrientes en venta}

En esta cuenta se contabilizan los resultados de las ventas de inmovilizado material y de las participaciones. También hay sólo dos años. El resultado es positivo y además se incrementaron siendo el $2.13 \%$ en 2008 de la suma de saldo de las participadas y el inmovilizado material y el 3.01\% en 2009 .

No existen diferencias entre bancos y cajas, ni correlación por tamaño.

\section{G.- Ganancias (pérdidas) de activos no corrientes en venta no clasificados como operaciones interrumpidas}

En esta cuenta se contabilizan los resultados por la venta de los activos no corrientes en venta, (inmuebles en dación de pago o adjudicados) las dotaciones por deterioro por adjudicaciones y los resultados por baja de inversiones estratégicas.

En este caso se ha pasado de unas ganancias del $22.19 \%$ sobre esta cuenta a unas pérdidas del $2.56 \%$ sobre la base de los activos no corrientes en venta.

Este cambio se ha producido tanto en bancos como en cajas, en los bancos se ha pasado del $17.13 \%$ al $-4.07 \%$ y en las cajas del $37.46 \%$ al $-1.13 \%$.

Por tamaño se observan dos grupos diferenciados. En las grandes y muy grandes la evolución ha sido similar de unos beneficios importantes en 2008 a unas pérdidas moderadas en 2009. En el resto de entidades, medianas, pequeñas

\footnotetext{
${ }^{3}$ Tras el cambio de formato de las cuentas anuales sólo aparece desde 2008.
} 
y muy pequeñas los resultados eran menores en 2008 y han disminuido en menor medida en 2009.

\subsection{Rentabilidades}

En las rentabilidades es donde más se puede comprobar los efectos de la crisis financiera, así como las diferencias entre los diferentes grupos estudiados.

\section{A.-Beneficios antes de impuestos sobre activo}

La rentabilidad sobre el activo (ROA) se ha reducido a la mitad en estos cuatro años, en 2006 era el $1.25 \%$ y en 2009 el $0.69 \%$.

La diferencia en esta disminución es muy diferente entre los bancos y las cajas. En 2006 tenían unas rentabilidades muy similares, los bancos, el 1.27\% y las cajas, el $1.20 \%$. El descenso se ha producido en los dos grupos de entidades, pero mucho más acentuado en las cajas. En los bancos en 2009 el ROA había descendido hasta el $0.84 \%$, dos tercios del que tenía en 2006, pero en las cajas esta disminución llevó al ROA al $0.36 \%$, un tercio del que tenían en 2006.

Existe una correlación entre tamaño y ROA, cuanto menor son las entidades menor es su ROA y más acentuado ha sido su descenso. La rentabilidad media de las muy grandes ha sido del $1.09 \%$, de las grandes del $0.73 \%$, de las medianas del $0.62 \%$, de las pequeñas del $0.77 \% \mathrm{y}$ de las muy pequeñas del $0.54 \%$.

\section{B.-Beneficios antes de impuestos sobre fondos propios}

La tendencia es similar en cuanto a la rentabilidad sobre fondos propios (ROE). Se ha pasado de un ROE del 20.29\% en 2006 al 11.16\% en 2009.

La caída ha sido mucho mayor en las cajas que en los bancos, además de partir de unas rentabilidades menores. En los bancos se pasó de un ROE del $22.68 \%$ en 2006 al $14.45 \%$ en 2009 , con una rentabilidad media ponderada del $20.37 \%$. En las cajas se ha pasado de un ROE del $17.21 \%$ en 2006 al $5.67 \%$ en 2009 con una rentabilidad media del $10.37 \%$.

También existe correlación entre rentabilidad y tamaño, cuanto más pequeñas son las entidades menor es su rentabilidad y más ha disminuido. Las muy grandes obtuvieron una rentabilidad media ponderada en este periodo del $19.37 \%$, las grandes del $13.92 \%$, las medianas del $8.80 \%$, las pequeñas del $10.37 \%$ y las muy pequeñas del $8.94 \%$.

\section{C.- Beneficios del grupo sobre activo}

La tendencia de disminución en cuanto a las rentabilidades del grupo es similar a la de las entidades sin consolidar, pero las rentabilidades del grupo son menores que las de las entidades sin consolidar, lo que significa que las entida- 
des dependiente obtienen menores rentabilidades que las matrices. El ROA del grupo en 2006 era del 1.97\% y en 2006 era la mitad el $0.44 \%$.

El ROA ya era menor en las cajas que en los bancos en 2006 y además ha disminuido en mayor medida en este periodo. En los bancos en 2006 era del $1.01 \%$ y en 2009 del $0.53 \%$ la mitad. En las cajas era del $0.89 \%$ en 2006 y del $0.29 \%$ en 2009 .

Existe una clara correlación entre el tamaño de la entidad y el ROA, cuanto menor son las entidades menor es el ROA y más ha disminuido en este periodo. La rentabilidad media de las entidades muy grandes ha sido del $0.78 \%$, de las grandes el $0.58 \%$, de las medianas el $0.52 \%$, de las pequeñas el $0.53 \%$ y de las muy pequeñas del $0.42 \%$.

\section{D.-Beneficios del grupo Sobre fondos propios}

E1 ROE en este periodo ha bajado de forma importante, del $19.09 \%$ que tenían por término medio todas las entidades, hasta el $7.65 \%$ que tenía en 2009 , menos de la mitad.

Aunque tanto cajas como bancos disminuyen el ROE, en las cajas es más pronunciado, además de partir de unas rentabilidades menores. Los bancos tenían un ROE en 2006 del $20.70 \%$ y en 2009 del $9.54 \%$, la mitad. Las cajas en 2006 tenían un ROE del $16.72 \%$ y en 2009 del $5.22 \%$, un tercio del que tenían en 2006.

La correlación entre tamaño y ROE, también existe, cuanto menor es la entidad menor es su ROE, las muy grandes alcanzaron el $14.83 \%$, las grandes, el $12.39 \%$, las medianas el $8.54 \%$, las pequeñas el $8.49 \%$ y las muy pequeñas el $6.99 \%$.

\section{RESULTADOS}

Con los datos de los cuatro años que se han analizando se elaboran los indicadores para evaluar de forma cualitativa, a los distintos grupos, (cajas y bancos, y tamaño) en tres modelos, ingresos, gastos y rentabilidades.

Cada uno de los tres modelos se construye con los datos de los indicadores expuestos en el punto 3 y para la valoración global los ponderamos por la ratio entre el valor de la masa patrimonial que representan, sobre la suma de todas.

\section{A.- Ingresos}

La ponderación de cada indicador para calcular la valoración global de los ingresos, se ha calculado como el porcentaje del valor absoluto de los datos que representa el indicador, sobre el total de los ingresos. En este caso los indicadores que más ponderan son el margen bruto, el margen de intermediación y las comisiones. 
Vemos que los bancos obtienen mejor valoración en todos los indicares que las cajas, excepto en los resultados de operaciones financieras. Además con diferencias muy significativas.

Por tamaño existen diferencias, aunque no siempre sigue el orden de mayor a menor o viceversa.

En el margen de intermediación el mejor resultado es el de las muy pequeñas, seguido de las muy grandes y el peor el de las grandes. En las comisiones sí que existe correlación entre tamaño y valoración, cuanto más grandes mejor valoración. También ocurre lo mismo con los resultados de las participadas, aunque en este caso existe una excepción con las pequeñas. (Ver Tabla $n^{\circ} 2$ ).

En cuanto a la valoración global del indicador de ingresos, el grupo que tienen mejor valoración son las muy grandes, sin embargo la peor valoración corresponde a las grandes. El segundo grupo con mejor valoración son las muy pequeñas y las medianas y pequeñas obtiene mejor valoración que las grandes pero peor que las muy pequeñas.

Por lo tanto vemos que en cuanto a los ingresos la diferencia entre bancos y cajas es muy notable. Sin embargo aunque sí que existen diferencias por tamaño, estas diferencias no siguen una correlación respecto al tamaño, ya que las muy pequeñas obtienen unos resultados mejores que todas, excepto las muy grandes.

Según los datos obtenidos sería positivo que las cajas se gestionaran como los bancos, pero no estaría tan claro que mejoraran los ingresos por aumentar de tamaño.

Tabla 2

Indicadores de ingresos

\begin{tabular}{|c|c|c|c|c|c|c|c|}
\hline & $\begin{array}{c}\text { IMi Margen de } \\
\text { intermediación }\end{array}$ & $\begin{array}{c}\text { IRc } \\
\text { Rendimientos } \\
\text { instrumentos } \\
\text { de capital }\end{array}$ & $\begin{array}{c}\text { IRp } \\
\text { Resultado } \\
\text { participadas }\end{array}$ & $\begin{array}{c}\text { Ic } \\
\text { Comisiones }\end{array}$ & $\begin{array}{c}\text { IRop } \\
\text { Resultados } \\
\text { de } \\
\text { operaciones } \\
\text { financieras }\end{array}$ & $\begin{array}{c}\text { IMb } \\
\text { Margen } \\
\text { bruto }\end{array}$ & cualificación \\
\hline Ponderación & 0,31 & 0,01 & 0,01 & 0,11 & 0,05 & 0,51 & 1,00 \\
\hline BANCOS & 0,290 & 1,460 & 1,330 & 0,150 & $-0,010$ & 0,180 & 0,229 \\
\hline CAJAS & $-0,270$ & $-0,650$ & $-0,970$ & $-0,220$ & 0,020 & $-0,420$ & $-0,338$ \\
\hline MUY GRANDES & 0,090 & 0,830 & 0,940 & 0,120 & $-0,010$ & 0,280 & 0,203 \\
\hline GRANDES & $-0,270$ & $-0,390$ & $-2,610$ & $-0,180$ & $-0,070$ & $-0,490$ & $-0,385$ \\
\hline MEDIANAS & $-0,210$ & $-0,780$ & $-3,640$ & $-0,290$ & 0,130 & $-0,170$ & $-0,221$ \\
\hline PEQUEÑS & $-0,080$ & $-1,610$ & 4,620 & $-0,240$ & $-0,040$ & $-0,290$ & $-0,179$ \\
\hline MUY PEQUENAS & 0,410 & $-0,960$ & $-9,680$ & $-0,220$ & $-0,160$ & $-0,090$ & $-0,052$ \\
\hline
\end{tabular}

Fuente: Elaboración propia. 


\section{B.- Gastos}

En el modelo para la valoración de los gastos, la ponderación la hemos calculado de igual manera, pero los dos indicadores de eficiencia los hemos considerado como sólo uno, asignado la mitad de ponderación a cada uno.

En los gastos los bancos no obtienen mejor valoración que las cajas en todos los indicadores, como ocurría en los indicadores de ingresos, ni las diferencias son tan grandes. En este caso los bancos obtienen mejor valoración que las cajas en la eficiencia medida sobre el margen bruto, pero no cuando la medimos sobre el total del activo, donde son las cajas las que obtienen mejor valoración. En este periodo, los bancos también obtienen peor valoración en las pérdidas por deterioro de inversiones crediticias, en las pérdidas de otros instrumentos financieros y en los resultados de la venta inmovilizado material, resultados de las participadas, venta de los inmuebles adjudicados y venta de inversiones estratégicas. Obtienen mejor valoración en el resultado de explotación y en la pérdida por deterioro del resto de activos, donde se contabilizan las dotaciones para pérdidas en el fondo de comercio, el deterioro de las acciones de las participadas y el deterioro en existencias, (solares, promociones en curso y construcciones y edificios).

Por tamaño, en la eficiencia sobre activo las muy pequeñas y las grandes son las que obtienen mejor valoración, y las muy grandes y medianas la peor. Sin embargo en la eficiencia sobre margen bruto son las muy grandes y las grandes las que obtienen la mejor valoración, siendo las muy pequeñas y las medianas la peor valoración. En cuanto a dotaciones para las inversiones crediticias la mejor valoración es la de las muy pequeñas y grandes, y la peor la de las muy grandes y medianas. En el resultado de explotación son las muy grandes la que obtienen mejor valoración y las muy pequeñas y medianas la peor. (Ver Tabla $n^{\circ} 3$ )

En el cálculo global del modelo de gastos, los bancos obtienen mejor valoración que las cajas, aunque las diferencias son menores que en el modelo de ingresos.

Por tamaño si que existen diferencia, pero estas diferencias no se corresponden de mayor a menor. Ya que la que obtiene mejor valoración es el grupo de las muy grandes, después la de las pequeñas, la tercera valoración mejor es la de las muy pequeñas, después la de las medianas, y la peor valoración la de las grandes.

Por lo tanto vemos que en este caso también es acertada la decisión de cambio de gestión de las cajas para que se gestionen como los bancos. Pero en cuanto a tamaño no está tan claro, ya que si bien las entidades muy grandes son las que mejor valoración obtienen, las grandes son las que peor valoración tienen. 
Tabla 3

Indicadores de gastos

\begin{tabular}{|c|c|c|c|c|c|c|c|c|c|}
\hline & $\begin{array}{c}\text { IEa } \\
\text { Eficiencia } \\
\text { sobre activo } \\
\text { (Gastos de } \\
\text { adminis- } \\
\text { tración/activo) }\end{array}$ & $\begin{array}{c}\text { IEMb } \\
\text { Eficiencia } \\
\text { Gastos de } \\
\text { administración } \\
\text { /margen bruto }\end{array}$ & $\begin{array}{c}\text { IPic } \\
\text { Pérdidas } \\
\text { por } \\
\text { deterioro } \\
\text { de inver- } \\
\text { siones } \\
\text { crediticias }\end{array}$ & \begin{tabular}{|c|} 
IPpyg \\
Pérdidas \\
otros ins- \\
trumentos \\
financieros \\
no valora- \\
dos a \\
valor \\
razonable \\
con \\
cambios \\
en p y g \\
\end{tabular} & $\begin{array}{c}\text { IRe } \\
\text { Resultado } \\
\text { de ex- } \\
\text { plotación }\end{array}$ & $\begin{array}{l}\text { IPra } \\
\text { Pérdidas } \\
\text { por } \\
\text { deterioro } \\
\text { del resto } \\
\text { de } \\
\text { activos }\end{array}$ & $\begin{array}{c}\text { IGre } \\
\text { Ganancias } \\
\text { (pérdidas) } \\
\text { en la baja } \\
\text { de activos } \\
\text { no clasifi- } \\
\text { cados } \\
\text { como no } \\
\text { corrientes } \\
\text { en venta }\end{array}$ & $\begin{array}{c}\text { IGAncv } \\
\text { Ganancias } \\
\text { (pérdidas) de } \\
\text { activos no } \\
\text { corrientes en } \\
\text { venta no } \\
\text { clasificados } \\
\text { como opera- } \\
\text { ciones } \\
\text { interrumpidas }\end{array}$ & $\begin{array}{l}\text { Cualifi- } \\
\text { cación }\end{array}$ \\
\hline Ponderación & 0,168 & 0,168 & 0,18 & 0,16 & 0,25 & 0,03 & 0,02 & 0,01 & 1,00 \\
\hline BANCOS & $-0,090$ & $-0,050$ & $-0,060$ & $-0,150$ & 0,120 & 13,970 & $-0,080$ & $-1,970$ & 0,437 \\
\hline CAJAS & 0,100 & $-2,540$ & 0,100 & 0,120 & $-0,210$ & 3,650 & 0,020 & 5,010 & 0,204 \\
\hline M. GRANDES & $-0,060$ & 1,250 & $-0,090$ & 0,130 & 0,140 & 2,020 & $-0,960$ & 2,380 & 0,113 \\
\hline GRANDES & 0,240 & 0,740 & 0,170 & $-0,530$ & $-0,170$ & $-11,100$ & 0,340 & 0,110 & $-0,434$ \\
\hline MEDIANAS & $-0,030$ & $-3,750$ & $-0,010$ & $-0,100$ & $-0,270$ & $-1,880$ & 0,610 & $-7,860$ & $-0,258$ \\
\hline PEQUEÑAS & 0,050 & $-1,940$ & 0,150 & 0,230 & $-0,090$ & 3,370 & 0,920 & $-8,640$ & 0,062 \\
\hline M. PEQUEÑAS & 0,490 & $-17,860$ & 0,320 & $-0,030$ & $-0,360$ & $-2,160$ & 0,490 & $-7,900$ & $-0,134$ \\
\hline
\end{tabular}

Fuente: Elaboración propia.

\section{C.- Rentabilidades}

Las rentabilidades las ponderamos las cuatro por igual, al 25\%.

En cuanto a las rentabilidades los bancos obtienen mejor rentabilidad en todos los indicadores y además de forma notable.

En cuanto a tamaño, en este caso tampoco se da una correlación entre el tamaño y rentabilidades en todos los indicadores, aunque sí que es mayor que en los otros modelos, cuanto mayor es el tamaño mayor es la rentabilidad. (Ver Tabla $\left.n^{\circ} 4\right)$.

En cuanto a la valoración global del indicador de rentabilidad existe una notable diferencia entre bancos y cajas. La valoración que obtienen los bancos en este modelo es muy superior a la de las cajas.

En este caso las muy grandes son las que mejor valoración tienen seguida de las grandes, y las pequeñas. Y las que peor valoración tienen son las medianas y las muy pequeñas.

En este caso vemos que también es acertada la decisión de cambio de gestión para las cajas, y en cuanto al tamaño vemos en este caso que también las entidades grandes y muy grandes son las que obtienen mejores rentabilidades. 
Tabla 4

Indicadores de rentabilidad

\begin{tabular}{|c|c|c|c|c|c|}
\hline & $\begin{array}{c}\text { IROAe } \\
\text { ROA ENTIDAD }\end{array}$ & $\begin{array}{c}\text { IROEe } \\
\text { ROE } \\
\text { ENTIDAD }\end{array}$ & $\begin{array}{c}\text { IROAg } \\
\text { ROA } \\
\text { GRUPO }\end{array}$ & $\begin{array}{c}\text { IROEg } \\
\text { ROE } \\
\text { GRUPO }\end{array}$ & CUALIFICACION \\
\hline Ponderación & 0,25 & 0,25 & 0,25 & 0,25 & 1.00 \\
\hline BANCOS & 0,130 & 3,960 & 0,080 & 1,600 & 1,443 \\
\hline CAJAS & $-0,250$ & $-6,040$ & $-0,140$ & $-4,030$ & $-2,615$ \\
\hline MUY GRANDES & 0,160 & 2,960 & 0,090 & 4,810 & 2,005 \\
\hline GRANDES & $-0,200$ & $-2,490$ & $-0,110$ & $-2,440$ & $-1,310$ \\
\hline MEDIANAS & $-0,300$ & $-7,610$ & $-0,170$ & $-3,850$ & $-2,983$ \\
\hline PEQUEÑAS & $-0,160$ & $-6,040$ & $-0,160$ & $-0,050$ & $-1,603$ \\
\hline MUY PEQUEÑAS & $-0,390$ & $-7,470$ & $-0,270$ & $-1,500$ & $-2,408$ \\
\hline
\end{tabular}

Fuente: Elaboración propia.

\section{D.- Total}

El último modelo en donde incluimos las valoraciones de los tres modelos anteriores se pondera cada uno al $33 \%$, ingresos, gastos y rentabilidades.

Vemos que la valoración de los bancos es muy superior a la de las cajas sobre todo en rentabilidades, también en ingresos y algo menor en gastos.

Por tamaño la mejor valoración en los tres modelos es la de las muy grandes. Pero las grandes no son las que obtienen la segunda mejor valoración en los tres, ya que sólo lo obtienen en rentabilidad, siendo las muy pequeñas las que obtienen la segunda mejor valoración en ingresos, y las pequeñas en gastos. La peor valoración la obtienen las grandes y las medianas en ingresos y gastos, y las medianas y muy pequeñas en rentabilidad. (Ver Tabla $n^{\circ} 5$ ).

Tabla 5

Valoración global

\begin{tabular}{|c|c|c|c|c|}
\hline & INGRESOS & GASTOS & RENTABILIDAD & CUALIFICACION \\
\hline Ponderación & 0,33 & 0,33 & 0,33 & \\
\hline BANCOS & 0,229 & 0,437 & 1,443 & 0,703 \\
\hline CAJAS & $-0,338$ & 0,204 & $-2,615$ & $-0,916$ \\
\hline MUY GRANDES & 0,203 & 0,113 & 2,005 & 0,774 \\
\hline GRANDES & $-0,385$ & $-0,434$ & $-1,310$ & $-0,710$ \\
\hline MEDIANAS & $-0,221$ & $-0,258$ & $-2,983$ & $-1,154$ \\
\hline PEQUEÑAS & $-0,179$ & 0,062 & $-1,603$ & $-0,573$ \\
\hline MUY PEQUEÑAS & $-0,052$ & $-0,134$ & $-2,408$ & $-0,865$ \\
\hline
\end{tabular}

Fuente: Elaboración propia. 
En la valoración global los bancos han gestionado de forma mucho más eficiente la actual crisis financiera que las cajas.

Por tamaño las entidades muy grandes son las que mejores resultados obtienen, sin embargo las grandes no es el segundo mejor grupo, ya que este puesto lo obtiene las pequeñas, en tercer lugar las grandes, después las muy pequeñas y la peor valoración la obtienen las medianas.

\section{CONCLUSIONES}

Por los resultados obtenidos se ha comprobado que la gestión de las entidades financieras en el periodo estudiado, ha sido muy diferente entre los bancos y las cajas de ahorros. Los bancos han sabido gestionar mejor este periodo de crisis financiera y aunque han sufrido las consecuencias de la actual crisis, las consecuencias no han sido tan graves como para las cajas de ahorro.

Como puntos fuertes de los bancos respecto de las cajas destaca las cuentas de ingresos. Los bancos han aumentado de forma significativa el margen de intermediación respecto de las cajas y también han incrementado de forma significativa los ingresos en concepto de comisiones, mientras que las cajas los han disminuido y sin embargo los bancos han aumentado los depósitos de los clientes cuatro veces más que las cajas. La política de selección de inversiones, tanto en instrumentos de capital como de empresas participadas, ha sido mucho más acertada en los bancos que en las cajas, muestra de ello es que la rentabilidad obtenida en los bancos casi dobla a la obtenida por las cajas.

No existe tanta diferencia entre bancos y cajas en las cuentas de gastos. Los ratios de eficiencia son bastante similares, si bien en los bancos son mejores respecto a margen bruto y en las cajas son mejores si los medimos sobre activo. En cuanto al conjunto de las dotaciones por deterioro, las cajas alcanzan unos resultados peores que los bancos, posiblemente a causa del alto riesgo inmobiliario, ya que la inversión en activos inmobiliarios es casi el triple, respecto del total del activo, en las cajas que en los bancos.

La rentabilidad, que ya era mejor en los bancos que en las cajas, ha incrementado el diferencial llegando ha ser un 70\% mayor el de los bancos que el de las cajas. A pesar de que tanto en cajas como en bancos la rentabilidad se ha visto muy deteriorada en este periodo.

No existe una clara relación entre tamaño y adecuación a la crisis en el periodo estudiado. Si bien las entidades muy grandes sí que son las que obtienen unos mejores resultados en este periodo, no pasa lo mismo con el siguiente grupo, las grandes, ya que este grupo obtiene los peores resultados de los cinco grupos formados. Hay que tener en cuenta que en este grupo formado por 8 entidades, esta la CAM, Caja Cataluña, Caja Galicia, entidades intervenidas por 
el Banco de España, y Bancaja. Por lo tanto en este grupo se ha concentrado muchos de los problemas de la actual crisis financiera.

Por lo tanto según los resultados obtenidos la posición del Banco de España en cuanto a orientar la restructuración financiera hacia la bancarización de las cajas es adecuada, en la gestión de la crisis financiera de 2006 a 2009. Luego es deseable que se profundice en el proceso de bancarización de las cajas de ahorros que se esta llevado ha cabo.

No está tan clara la ventaja que se obtendrá por aumentar el tamaño, ya que si bien las entidades más grandes son las que mejores resultados obtienen no es así con las grandes, y sin embargo las pequeñas obtienen mejores resultados globales que las medianas y que las grandes. También se debe de tener en cuenta, que en el actual proceso de fusiones, existen dos diferencias sustanciales, las fusiones interregionales y la bancarización, por lo que será un buen tema de investigación cómo evolucionan estas entidades en el futuro.

\section{REFERENCIAS BIBLIOGRÁFICAS}

APELLANIZ, P; SERRANO, C; Y APELLANIZ, T. (1995). El impacto de las fusiones en el sector de las cajas de ahorros españolas. VII Congreso de Aeca, septiembre 1995.

ARÉVALO QUIJADA, T; GÓMEZ DOMÍNGUEZ, D; VÁZQUEZ CUETO, J; ZAPATA REINA, A. (2002). Un estudio de las cajas de ahorros andaluzas mediante el método multicriterio Promethee. Estudios de Economía Aplicada, vol. $20 n^{\circ} 1$ abril 2002

AZOFRA PALENZUELA, V.; SANTAMARÍA MARISCAL, M. (2004) El gobierno de las cajas de ahorro españolas. Universia Business Review, segundo trimestre, número 0022004

COELLO ARANDA, J. (1994). ¿son las cajas y los bancos estratégicamente equivalentes? Investigaciones económicas. Vol. XVIII mayo 1994

COLARTE MORANDO, T. E. B.; RODRÍGUEZ FERNÁNDEZ, J. M. (2006). Resultados económicos y financieros de las fusiones en España: un Análisis Empírico con información contable. Revista Española de Financiación y Contabilidad. Vol XXXV, n 129 abril junio 2006.

FONSECA DÍAZ, A. R.; FERNÁNDEZ RODRÍGUEZ, E.; MARTÍNEZ ARIAS, A. (2011). Factores condicionantes de la presión fiscal de las entidades de crédito españolas. ¿Existen diferencias entre bancos y cajas de ahorros? Revista española de Financiación y Contabilidad. Vol XXXIX $n^{\circ} 152$ julioseptiembre 2011 pp 491-516. 
GILBERT, R. A, (1984). Bank Market Structure and Competition. Jornal of money, Credit and banking v. $16 n^{\circ} 4$ November

GONZÁLEZ-PÁRAMO, J. M. (2011). La Estabilidad financiera en la zona del euro y el riesgo sistémico. Información Comercial Española. $N^{\circ} 863$ noviembre-diciembre 2011.

GUAL, J.; HERNANDEZ, A. (1991). Costes operativos, tamaño, y especialización en las cajas de ahorro españolas. Investigaciones económicas. Vol $X V n^{\circ} 3$

GUERRERO MORA, R.; VILLALPANDO BENITTEZ, M. (2009). Rentabilidad, concentración y eficiencia en el sistema bancario mexicano. El Trimestre Económico vol LXXVI México Enero - Mazo.

JENSEN, M.C. y MECKLING, N.H. (1976): Theory of the firm: Managerial behavior, agency costs and ownership structure. Journal of FinancialEconomics, N. 3,

MARÍN, S.; GÓMEZ, J.; CÁNDIDO GÓMEZ, J. (2008). Eficiencia técnica en el sistema bancario español. Dimensión y rentabilidad. El Trimestre Económico. Vol LXXV México Octubre - Diciembre pp. 1017-1042

MARTINEZ ARIAS, A. (1998). Las fusiones de Cajas españolas: Un estudio empírico. Revista Española de Financiación y Contabilidad. Vol XXVII, n $n^{\circ} 97$ octubre-diciembre 1998.

MAUDOS, J. Y PÉREZ, F. (2003): "Competencia vspoder de mercado en la bancaespañola", Moneda y Crédito Vol. 217, pp. 139-166

PALOMO ZURDO, R. J. Y SANCHÍS PALACIO, J. R. (2010). Efectos de las fusiones sobre la concentración y la eficiencia bancaria: El caso de las cajas rurales y los retos de la crisis financiera. Revista Española de Financicion y Contabilidad Vol. XXXIX abril junio 2010

PASTOR, J.M. (1995): Eficiencia, cambio productivo y cambio técnico en los bancos y cajas de ahorros españolas: un análisis de la frontera no paramétrico. Revista Española de Economía, Vol. 12, N. 1

SMIRLOCK M. (1985). Evidence on the (non) relationship between concentration and profitability in banking. Journal of Money, Credit and Banking. Vol $17 \mathrm{n}^{\circ} 1$ february.

VIVES, X. (2011). El paradigma de la competencia en el sector bancario después de la crisis. Papeles de Economía Española, nº 130, 2011. 